Pensamiento Crítico N. ${ }^{\circ} 11$, pp. $129-138$

\title{
Análisis de riesgos en proyectos de inversión
}

\author{
Elías Sánchez Z.
}

\section{RESUMEN}

En casi todos los proyectos privados utilizan conceptos como demanda, oferta, aversión al riesgo, etc.; sin embargo, pocas veces observamos la aplicación de instrumentos propios del análisis económico para evaluar estos aspectos. Mi experiencia profesional me permite verificar la importancia del instrumental económico al medir el impacto del grado de aversión al riesgo del decisor, en particular, y el análisis de riesgos, en general. Ante escasos recursos, alta incertidumbre y escasas alternativas de elección elevan la certeza en las decisiones; sin embargo, pocas veces son utilizados por su "complicada" aplicación, percepción que pretendemos reducir con el presente artículo.

Palabras clave: Decisiones, proyectos, valor actual neto, incertidumbre, riesgo, aversión al riesgo.

\section{ABSTRACT}

In almost all private projects, we use concepts like demand, supply, risk aversion, etc. although, just a few times, we observes the application of instruments properly related to the economic analysis to evaluate these projects. The experiences obtained in my professional life let me verify the importance of the economic instruments 


\section{Elías D. Sánchez Z.}

for measuring the impact of the degree of aversion to the risk decision-maker in particular, and the risk analysis, in general. To scarce resources, high uncertainty and few alternative of election, there is a high certainty in the decisions; however, they are rarely used for their "complicated" aplication, perception that we intend to reduce with this article.

Keywords: Decisions, projects, net present value, uncertainty, risk, risk aversion.

\section{DE LOS CRITERIOS DE LOS INVERSIONISTAS}

Para evaluar un proyecto de inversión es usual recurrir al criterio de valor actual neto (VAN), que permite determinar si en un horizonte temporal definido la suma actualizada de los márgenes de rentabilidad proyectados es mayor que la inversión a realizar.

Sin embargo, la existencia de incertidumbre respecto al futuro hace que la previsión de los flujos proyectados sea imperfecta; este aspecto "avisa" que enfrentamos riesgos de no obtener el VAN calculado (el VAN resulta un valor esperado, ¿cuán probable es que ocurra este valor o uno cercano?).

Ante la posibilidad de perder, es necesario realizar análisis de riesgos. El riesgo se expresa en la variabilidad del VAN (una mayor variación implica mayor riesgo de no obtener el VAN esperado). Debe recordarse que un mayor riesgo (mayor incertidumbre) está asociado a mayor rentabilidad ${ }^{1}$.

Por ejemplo, suponga un VAN de 10000 u.m., cinvertiría si fluctúa entre 9000 u.m. y 11000 u.m.?, ¿invertiría si fluctúa entre -30000 u.m. y 50000 u.m.? La segunda opción podría otorgar una ganancia mayor pero también mayor pérdida: opciones con igual VAN presentan riesgos distintos.

Para analizar la variabilidad o riesgo del VAN, existen muchas metodologías, algunas explicativas (¿jpor qué surgen los riesgos?) y otras descriptivas (¿̇uál es el impacto de los riesgos?). Nosotros nos centraremos en evaluar el impacto de los riesgos en el VAN (métodos descriptivos, propios para decisiones en proyectos).

1 Al respecto, la gestión del riesgo reconoce lo siguiente: “... si una empresa está posicionada estratégicamente para tomar ventaja de las fluctuaciones del VAN, existe valor en la incertidumbre" (Decisioneering). 


\section{Análisis de riesgos en proyectos de inversión}

\section{LOS MÉTODOS EN EL ANÁLISIS DE LOS PROYECTOS DE INVERSIÓN}

Utilicemos un ejemplo para exponer la forma en la cual se utilizan estos métodos en los proyectos de inversión. Suponiendo que la evaluación económico financiera de un proyecto individual (con una inversión de US\$ 12 millones) muestre el siguiente resumen de resultados:

\begin{tabular}{|lccccc|}
\hline \multicolumn{7}{|c|}{$\begin{array}{c}\text { Resumen resultados } \\
\text { (En miles de dólares) }\end{array}$} \\
\hline & $\mathbf{2 0 0 8}$ & $\mathbf{2 0 0 9}$ & $\mathbf{2 0 1 0}$ & $\mathbf{2 0 1 1}$ & $\mathbf{2 0 1 2}$ \\
\hline EBIT & $4,734.80$ & $5,169.31$ & $5,623.37$ & $6,097.87$ & $6,593.72$ \\
Depreciación & $2,299.00$ & $2,402.46$ & $2,510.57$ & $2,623.54$ & $2,741.60$ \\
EBITDA & $7,033.80$ & $7,571.77$ & $8,133.94$ & $8,721.41$ & $9,335.32$ \\
Ctas cobrar & $3,831.67$ & $4,004.09$ & $4,184.28$ & $4,372.57$ & $4,569.33$ \\
Ctas pagar & $4,563.80$ & $4,713.81$ & $4,870.57$ & $5,034.38$ & $5,205.57$ \\
FC Operativo & 5139.88 & 5504.04 & 5884.59 & 6282.26 & 6697.83 \\
Gastos capital & $1,000.00$ & $1,000.00$ & $1,000.00$ & $1,000.00$ & $1,000.00$ \\
\hline FC Libre & $4,210.49$ & $4,018.67$ & $4,377.37$ & $4,752.22$ & $5,143.94$ \\
\hline Valor continuo & & & & & $29,848.00$ \\
FLC +VC & $4,210.49$ & $4,018.67$ & $4,377.37$ & $4,752.22$ & $34,991.94$ \\
VAN & $2,852.72$ & & & & \\
\hline
\end{tabular}

A nivel primario, el resultado sugiere que el proyecto debe realizarse dado que el VAN es positivo (US\$2.85 millones), pero esto ocurrirá solamente si no surgen variaciones en los valores estimados para calcularlo (es necesario identificar las variables críticas, las que determinan el éxito o el fracaso del proyecto).

\begin{tabular}{|c|c|c|c|}
\hline \multicolumn{4}{|c|}{ Variables críticas del proyecto - Sensibilidad del VAN } \\
\hline & Pesimista & Base & Optimista \\
\hline Ventas TM & $-1,812.73$ & & $7,518.17$ \\
\hline Precio & -540.33 & & $4,549.25$ \\
\hline Costos producción directos & $-1,440.80$ & & $4,692.80$ \\
\hline Costos producción indirectos & & & \\
\hline Gastos de ventas & $1,556.63$ & $2,852.72$ & $3,716.78$ \\
\hline Gasto admnistrativos & $2,503.71$ & & $3,085.39$ \\
\hline Días de cobranza & 959.61 & & $3,799.27$ \\
\hline Días de venta & $1,811.51$ & & $3,893.93$ \\
\hline Días de pago & $1,398.12$ & & $3,337.59$ \\
\hline
\end{tabular}




\section{Elías D. Sánchez Z.}

Las variables críticas son: ventas en unidades, precio y costo de producción. Este proceso se denomina análisis de sensibilidad, pero es incompleto porque solo considera el efecto del cambio de una variable crítica (no su probabilidad de ocurrencia y tampoco el impacto del cambio de más de una variable crítica).

Para evaluar el cambio en el VAN cuando varía más de una variable, así como la probabilidad de ocurrencia de dichos cambios recurrimos al análisis de escenarios, el cual consiste en definir un contexto de cambio en aspectos políticos, sociales, económicos, etc., y cómo estos alteran las variables críticas.

Asumiremos la existencia de potenciales cambios, tales como: (i) deterioro de los términos de intercambio para nuestro país; (ii) aparición competidores con tecnologías más eficientes; y/o, (iii) crecimiento económico sostenido con ingreso de capitales y reducción del riesgo país. Al respecto, los especialistas estiman que:

El deterioro de los términos de intercambio generaría caída de unidades vendidas en $15 \%$, aumento de precios en $8 \%$, aumento de costos de producción directos en $2 \%$ de las ventas, aumento de gastos fijos a US \$5,9 millones, aumento de días de cobranza a 90 días, días de venta a 100 días y días de pago a 120 días.

La entrada de competidores más eficientes generaría: caída de unidades vendidas en un $30 \%$, reducción de precios en $15 \%$, reducción de costos fijos a US $\$ 4,1$ (por reestructuración) y sin cambios en días de cobranza, de ventas y de pagos.

El crecimiento sostenido con entrada de capitales y reducción de riesgo país generaría: aumento de unidades vendidas en un $5 \%$ anual; aumento de precios en un $3 \%$, reducción de costos variables a $65 \%$ (mayor productividad) y mejora en días de venta a 45 días.

Cada uno de estos escenarios altera el VAN esperado. El deterioro de los términos de intercambio generaría una pérdida de US $\$ 5.54$ millones, la entrada de competidores eficientes una pérdida de US\$2.95 millones y el crecimiento sostenido prácticamente triplica el VAN (pasa a US\$ 7.96 millones). 


\section{Análisis de riesgos en proyectos de inversión}

Con esta información, ¿qué se decidiría, se haría el proyecto? Definitivamente, habría que preguntarse sobre la probabilidad de ocurrencia de cada escenario. Si existe alguna probabilidad de ocurrencia de estos escenarios, ¿Cuál será ahora el VAN esperado?

\begin{tabular}{|lcr|}
\hline \multicolumn{1}{|c}{ Escenario } & Probabilidad & VAN \\
\hline Caso base & $50 \%$ & $2,852.72$ \\
Deterioro de términos de intercambio & $20 \%$ & -5535.86 \\
Competidores más eficientes & $20 \%$ & -2950.53 \\
Crecimiento sostenido & $10 \%$ & 7960.67 \\
\hline \multicolumn{3}{c}{} \\
\hline VAN esperado & & 56502632 \\
Var (VAN) & & 6041.74 \\
DS (VAN) & & \\
\hline
\end{tabular}

La posible ocurrencia de los escenarios señalados $(50 \%, 20 \%, 20 \%$ y $10 \%)$ reduce el VAN esperado de US\$2.85 millones a US\$ 0.53 millones, con un rango de variación de más - menos US\$ 6.04 millones (el VAN podría estar entre los US\$ 5.52 millones de pérdida y los US\$ 6.57 millones de ganancia).

Estos escenarios reducen el VAN promedio (o esperado) a US $\$ 0.53$ y denotan un nivel importante de incertidumbre (por el rango de variabilidad) en el logro de un VAN positivo. En estos casos los tradicionales análisis de sensibilidad y de escenarios son insatisfactorios e insuficientes ${ }^{2}$.

Resulta necesario calcular la probabilidad de obtener algún VAN, es decir, debemos recurrir al método de simulación, el cual incorpora todo valor con posibilidad de realización (implícitamente considera todos los escenarios posibles) de cualquier variable (principalmente de las variables críticas).

Este método requiere: (i) establecer todos los valores y la frecuencia de ocurrencia de éstos para cada variable crítica; y, (ii) determinar la distribución de probabilidad del VAN con simulación de Montecarlo. Para el ejemplo se establece que:

2 Propios de escenarios muy estables (adecuados recursos, varias alternativas disponibles y baja incertidumbre), de bajo riesgo. 


\section{Elías D. Sánchez Z.}

- Las ventas se distribuyen normalmente $e^{3}$ con promedio igual al escenario base y con desviación estándar del 10\%. La venta mínima se estima no menor al $70 \%$ del valor base y existe correlación con el precio de -0.8 y correlación entre ventas de 0.9

- El precio de venta unitario se incrementará con la inflación y con el tamaño del mercado (el cual depende del PIB), por lo que éste se ubicará en un rango de S/.100 y S/. 120 pero con un valor más probable de S/. 110 (distribución triangular) ${ }^{4}$.

- Los costos de producción directos se distribuyen normalmente alrededor de un $48 \%$, con desviación estándar del 2\%. Mientras que los gastos fijos se ubicarán entre US\$ 5.7 y US \$ 4.3 millones con igual probabilidad de ocurrencia (distribución uniforme).

- La tasa de impuesto a la renta se mantendrá en $40 \%$, situándose entre $38 \%$ y $42 \%$ (distribución uniforme). Los días de cobranza, días de venta y días de pago presentarán valores promedio iguales a los del caso base con desviación estándar del $10 \%$ (distribución normal).

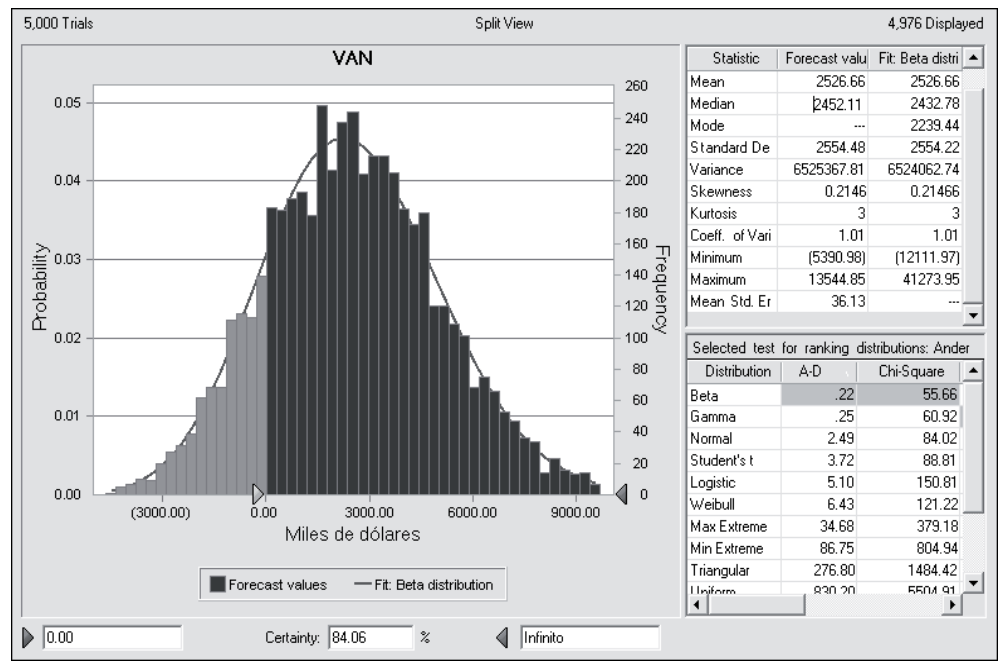

3 Apropiada para fenómenos recurrentes (rutinarios) y con valores concentrados simétricamente alrededor del valor promedio.

4 Utilizadas cuando se desconoce el comportamiento estadístico pero se cuenta con estadísticos como máximo, mínimo y moda. 


\section{Análisis de riesgos en proyectos de inversión}

La distribución de probabilidades del VAN resulta de las distribuciones de probabilidad señaladas. El método de simulación permite establecer que existe gran confianza (95\%) que el VAN será mayor a cero en 84 de cada 100 ocasiones que se realice lo establecido en el proyecto ( $16 \%$ de probabilidad de perder).

Es muy probable que el proyecto sea rentable (iexiste bajo riesgo de perder!). ¿Se decidiría hacer el proyecto? Considerando la simulación, son tres las variables críticas que contribuyen con el $94 \%$ de la varianza del VAN (cantidad vendida, gastos de ventas y precio).

\begin{tabular}{|c|c|c|c|}
\hline \multicolumn{2}{|c|}{1,000 Trials } & \multicolumn{2}{|l|}{ Sensitivity Data View } \\
\hline & Assumptions & Contribution to Variance & Rank Correlation \\
\hline D & "Ventas TM & $35.7 \%$ & 0.89 \\
\hline & ${ }^{x}$ Precio & $30.8 \%$ & 0.82 \\
\hline & "Gastos de ventas & $27.2 \%$ & -0.77 \\
\hline & Costos producción directo & $5.3 \%$ & -0.34 \\
\hline
\end{tabular}

La simulación permite establecer que la inversión de US\$12 millones, después de 5 años, puede generar entre US\$ 8.25 millones (pérdida máxima de US\$ 3.75 millones) y US\$23.25 millones (ganancia máxima US\$11.25 millones).

Los resultados extremos señalados son improbables, pero existen resultados intermedios que presentan mayor probabilidad de ocurrencia. Un analista financiero desarrollaría la siguiente tabla que muestra al decisor intervalos de posibles resultados con sus probabilidades de ocurrencia.

\begin{tabular}{|ccccc|}
\hline VAN (intervalo) & Probabilidad & VAN & VAN + Inversión & Resultado promedio \\
\hline Menor (3.0) & $1.8 \%$ & -3.75 & 8.25 & 0.15 \\
(3.0) a (1.5) & $5.4 \%$ & 2.25 & 9.75 & 0.52 \\
(1.5) a (0.0) & $12.0 \%$ & 0.75 & 11.25 & 1.35 \\
0.0 a 1.5 & $15.4 \%$ & 0.75 & 12.75 & 1.97 \\
1.5 a 3.0 & $16.9 \%$ & 2.25 & 14.25 & 2.40 \\
3.0 a 4.5 & $18.7 \%$ & 3.75 & 15.75 & 2.95 \\
4.5 a 6.0 & $12.9 \%$ & 5.25 & 17.25 & 2.23 \\
6.0 a 7.5 & $8.3 \%$ & 6.75 & 18.75 & 1.55 \\
7.5 a 9.0 & $5.3 \%$ & 8.25 & 20.25 & 1.08 \\
9.0 a 10.5 & $2.0 \%$ & 9.75 & 21.75 & 0.44 \\
Mayor a 10.5 & $1.3 \%$ & 11.25 & 23.25 & 0.30 \\
\hline \multicolumn{5}{c}{} \\
\hline
\end{tabular}




\section{Elías D. Sánchez Z.}

Basado en la tabla, el analista mostraría al decisor que el resultado esperado (VAN + Inversión esperados) con el proyecto es de US\$14.94 millones. La alternativa del decisor es comprar certificados de depósito al 5\% anual, en ese caso recibiría US\$13.87 millones a valor presente (deflactado al $2 \%$ anual).

La alternativa del proyecto podría otorgar US\$ 1.07 millones por encima de la opción de comprar certificados de depósito. En este caso, usualmente la recomendación sería no realizar el proyecto debido a que el proyecto presenta riesgos de perder, mientras que la compra de certificados de depósito es segura.

\section{EL COMPORTAMIENTO DEL DECISOR}

Aquí ingresamos a un escenario crucial del trabajo profesional, el cálculo probabilístico y financiero nos dice que no vale la pena asumir el riesgo (estamos asegurando la riqueza del inversionista); sin embargo, el inversionista podría pensar en forma distinta y apostar por ejecutar la inversión.

“... la evaluación de proyectos no debe ser clasificada como una técnica de toma de decisiones, sino sólo una herramienta que genera información [...] con la misma información, algunos inversionistas optan por aceptar destinar recursos para un proyecto, otros prefieren no hacerlo" 5

En ocasiones las personas nos arriesgamos para incrementar nuestro bienestar (consecuencias favorables) a niveles mayores de lo que podríamos perder (consecuencias desfavorables) con una apuesta. Es decir, buscamos asumir riesgos para ganar más, en lugar de tomar una alternativa segura.

Para aproximarnos a la decisión del decisor debemos evaluar su nivel de tolerancia al riesgo (establecer su grado de aversión al riesgo ${ }^{6}$ ). La microeconomía enseña a realizar esta medición, ¿Estará dispuesto nuestro decisor a asumir el riesgo de la inversión, será más atractivo para él invertir?

Si el decisor valora más las consecuencias favorables del proyecto respecto a las consecuencias desfavorables, estará más dispuesto a asumir el riesgo. Para deter-

5 Sapag, Nassir. Influencia del método de valor de desecho sobre la formulación del proyecto. www.nassirsapag.cl/art06.htm

6 Ver Mas-Collel et al. (1995). Microeconomic Theory, Choice under uncertainty, chapter 6, pp. 183-194. 


\section{Análisis de riesgos en proyectos de inversión}

minar esta valoración subjetiva definimos un índice de atractivo ${ }^{7}$ (100 para la mejor consecuencia, US $\$ 23.25$ millones, y 0 para la peor, US $\$ 8.25$ millones).

Las valoraciones se traducen en puntajes de atractivo y son obtenidas a través de entrevistas a profundidad o grupos focales. En este caso, el decisor asigna al proyecto un atractivo de 69.37 puntos (equivalente a US $\$ 14.28^{8}$ millones) mayor a los 66.14 puntos de los certificados (equivalente a US\$ 13.87 millones).

\begin{tabular}{|ccccc|}
\hline Probabilidad & VAN & VAN + Inversión & Puntaje Atractivo & Atractivo Promedio \\
\hline $1.8 \%$ & -3.75 & 8.25 & 0 & 0.00 \\
$5.4 \%$ & 2.25 & 9.75 & 25 & 1.34 \\
$12.0 \%$ & 0.75 & 11.25 & 46 & 5.52 \\
$15.4 \%$ & 0.75 & 12.75 & 59 & 9.10 \\
$16.9 \%$ & 2.25 & 14.25 & 71 & 11.97 \\
$18.7 \%$ & 3.75 & 15.75 & 78 & 14.62 \\
$12.9 \%$ & 5.25 & 17.25 & 84 & 10.84 \\
$8.3 \%$ & 6.75 & 18.75 & 92 & 7.60 \\
$5.3 \%$ & 8.25 & 20.25 & 96 & 5.12 \\
$2.0 \%$ & 9.75 & 21.75 & 98 & 1.96 \\
$1.3 \%$ & 11.25 & 23.25 & 100 & 1.31 \\
\hline $\mathbf{1 0 0 \%}$ & & & & $\mathbf{6 9 . 3 7}$ \\
\hline
\end{tabular}

El decisor ${ }^{9}$ valora el proyecto US\$ 0.41 millones por encima de los certificados de depósito; es decir, las consecuencias favorables de la inversión pesan más para él que las consecuencias desfavorables (el bienestar por ganar con la inversión es mayor al bienestar por perder).

El grado de aversión al riesgo del decisor de nuestro caso lo incentiva a ejecutar el proyecto. Alguien más adverso al riesgo podría valorar el proyecto por debajo de US\$ 13.87 millones, (no ejecutaría el proyecto). Si fuese neutral al riesgo valoraría la inversión en US\$14.94 millones (el resultado del análisis financiero) ${ }^{10}$.

7 La relación que define el índice es el equivalente a la correspondencia o función de utilidad en microeconomía.

8 Obtenido al resolver la inversa de la función o correspondencia de utilidad (estimada con la información de los cuestionarios).

9 Debemos considerar que la valoración de una persona es tan individual como su personalidad.

10 El análisis de las finanzas usualmente obvia las valoraciones personales en las decisiones (una razón de las crisis financieras). 


\section{Elías D. Sánchez Z.}

La valoración del proyecto para el decisor (US\$ 14.28 millones) es menor al cálculo financiero (US\$14.94 millones) debido a que tiene aversión al riesgo, pero mayor a la alternativa de certificados. La diferencia de US $\$ 0.66$ millones es una medida monetaria del grado de aversión al riesgo ${ }^{11}$.

Lo valioso de este análisis radica en la consideración de que no necesariamente un inversionista hará estricto caso a una recomendación financiera (que supone neutralidad al riesgo), la valoración personal de las consecuencias futuras de la apuesta podrían ser muy atractivas para el decisor.

Si el inversionista decide ejecutar la inversión, a pesar de la recomendación financiera, será necesario diseñar un programa que genere recomendaciones para lograr el máximo VAN posible dados los recursos, restricciones e información; es decir, un modelo de optimización (tema de un próximo artículo).

\section{BIBLIOGRAFÍA}

Decisioneering Inc. Crystal Ball 7. 2007, www.crystalball.com

Hammond, Keeney, Raiffa. Smart choice. Harvard Business School Press, Boston, USA, 1999.

Mas-Collel, Whinston, Green. Microeconomic Theory. Oxford University Press, New York, USA, 1995.

Sapag, Nassir. Influencia del método de valor de desecho sobre la formulación del proyecto, 2010, www.nassirsapag.cl/art06.htm

Sapag, Nassir. Preparación y evaluación de proyectos. McGraw Hill, Colombia, 2007.

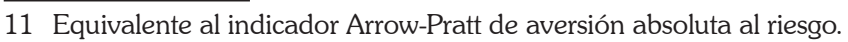

